\title{
Total Interval Number for Graphs with Bounded Degree
}

\author{
Alexander V. Kostochka ${ }^{1, *}$ \\ Douglas B. West ${ }^{2, \dagger}$ \\ 1 INSTITUTE OF MATHEMATICS, \\ RUSSIAN ACADEMY OF SCIENCES, \\ NOVOSIBIRSK, RUSSIA \\ E-mail address: sasha@math.nsc.ru \\ ${ }^{2}$ DEPARTMENT OF MATHEMATICS, \\ UNIVERSITY OF ILLINOIS, \\ URBANA, IL 61801-2975 \\ E-mail address:west@math.uiuc.edu
}

Received March 12, 1995

\begin{abstract}
The total interval number of an $n$-vertex graph with maximum degree $\Delta$ is at most $(\Delta+1 / \Delta) n / 2$, with equality if and only if every component of the graph is $K_{\Delta, \Delta}$. If the graph is also required to be connected, then the maximum is $\Delta n / 2+1$ when $\Delta$ is even, but when $\Delta$ is odd it exceeds $[\Delta+1 /(2.5 \Delta+7.7)] n / 2$ for infinitely many $n$. (c) 1997 John Wiley \& Sons, Inc. J Graph Theory 25: 79-84, 1997
\end{abstract}

Keywords: total interval number, intersection representation, maximum degree

Given sets $\left\{S_{v}: v \in V\right\}$, the intersection graph of the collection of sets is the simple graph with vertex set $V$ such that $u$ is adjacent to $v$ if and only if $S_{u} \cap S_{v} \neq \emptyset$. The family of sets is an intersection representation of its intersection graph. The interval graphs are the intersection graphs representable by assigning each vertex a single interval on the real line. More generally,

* Research supported in part by Grant 93-01-01486 of the Russian Foundation for Fundamental Research and Grant RPY300 of the International Science Foundation and Russian Government. † Research supported in part by NSA/MSP Grants MDA904-90-H-4011 and MDA904-93-H-3040.

c 1997 John Wiley \& Sons, Inc.

CCC 0364-9024/97/010079-06 
we allow a representation $f$ to assign each vertex a union of intervals on the real line; if $G$ is the intersection graph of this collection, then $f$ is a multiple-interval representation of $G$. Let \# $f(v)$ be the number of disjoint intervals whose union is $f(v)$. If $\# f(v)=k$, we say that $f(v)$ consists of $k$ intervals or that $v$ is assigned $k$ intervals.

We may try to make the representation of $G$ " "efficient" by minimizing $\max _{v \in V} \# f(v)$ or $\sum_{v \in V} \# f(v)$. The interval number $i(G)$ of a graph $G$ is the minimum of $\max _{v \in V(G)} \# f(v)$ over all multiple-interval representations of $G$. Interval number has been studied since 1979, beginning with [7] and [2]. The total interval number $I(G)$ of a graph $G$ is the minimum of $\sum_{v \in V(G)} \# f(v)$ over all multiple-interval representations of $G$. Although introduced in [2], total interval number was not studied until Aigner and Andreae [1] obtained extremal results for some fundamental families. Further results appear in $[3,4,5,6]$.

In this paper we prove that $I(G) \leq(\Delta+1 / \Delta) n / 2$ for graphs with maximum degree at most $\Delta$, which is best possible. The proof yields a polynomial algorithm for producing a representation that satisfies the bound. Kratzke and West [5] proved that if $G$ contains a collection of $t$ pairwise edge-disjoint trails that together contain an endpoint of every edge of $G$, then $I(G) \leq e(G)+t$, where $e(G)=|E(G)|$. Such a collection of trails is a trail cover of size $t$, generalizing the notion of vertex cover; in this paper, "covering $e$ "'means "containing an endpoint of $e$ ". Let $t(G)$ denote the minimum size of a trail cover. If $G$ is triangle-free, then $I(G)=e(G)+t(G)$; a simple counting argument [5] establishes the lower bound. The graph $m K_{\Delta, \Delta}$ has $m$ components that are complete bipartite graphs; it is regular and triangle-free, with $n=2 m \Delta$ vertices, and its minimum trail covers have size $m$. Hence $I\left(m K_{\Delta, \Delta}\right)=m \Delta^{2}+m=(\Delta+1 / \Delta) n / 2$, and our bound is best possible. We also prove that these are the only graphs achieving the bound. The proof yields a polynomial-time algorithm to achieve the bound.

\section{CONNECTED GRAPHS}

Before proving the bound for general graphs, we discuss the more restricted class of connected graphs. For $\Delta$ even, the maximum of $I(G)$ in terms of $n$ and $\Delta$ is $\Delta n / 2+1$. For $\Delta$ odd, we provide constructions where the excess over $\Delta n / 2$ is linear in $n$.

Proposition. Suppose $\Delta$ is even. Among connected $n$-vertex graphs with maximum degree $\Delta$, the maximum of the total interval number is $\Delta n / 2+1$.

Proof. Suppose $G$ is connected and has maximum degree $\Delta$. If $G$ is Eulerian, then $I(G) \leq$ $e(G)+1 \leq \Delta n / 2+1$, with equality if $G$ is triangle-free and regular. If $G$ is not Eulerian, suppose $G$ has $2 k$ vertices of odd degree. Since $G$ is connected, we can decompose $E(G)$ into $k$ trails, so $t(G) \leq k$. Since each vertex of odd degree has degree less than $\Delta$, we have $2 e(G) \leq \Delta n-2 k$, and hence $I(G) \leq e(G)+k \leq \Delta n / 2$.

In addition to the $\Delta$-regular triangle-free graphs, equality holds also for $\Delta$-regular graphs in which every vertex belonging to a triangle is a cut-vertex. When $\Delta$ is odd, the bound of $\Delta n / 2+1$ no longer holds; we provide a construction.

Proposition. Suppose $\Delta$ is odd and at least 3. Among connected $n$-vertex graphs with maximum degree $\Delta$, the maximum total interval number exceeds $[\Delta+1 /(2.5 \Delta+7.7)] n / 2$ for infinitely many $n$.

Proof. We prove the claim by using copies of a triangle-free graph $H$ with degree sequence $(\Delta, \ldots, \Delta, \Delta-1)$ to construct a $\Delta$-regular triangle-free connected graph $G$ with $n$ vertices such 
that $t(G) \geq[1 /(2.5 \Delta+7.7)] n / 2$. Begin with a caterpillar $C$ consisting of a path with $k+2$ vertices and $\Delta-2$ leaves attached to each interior vertex of the path. For each of the $k(\Delta-2)+2$ leaves of $C$, we provide a copy of $H$ and identify its vertex of degree $\Delta-1$ with that leaf.

The resulting graph $G$ is triangle-free and $\Delta$-regular, so $I(G)=\Delta n / 2+t(G)$. Because each edge of $C$ is a cut-edge of $G$, every trail cover of $G$ has an endpoint in each copy of $H$. Hence $t(G) \geq[k(\Delta-2)+2] / 2$, and in fact $t(G)=\lceil[k(\Delta-2)+2] / 2\rceil$. If $H$ has $n^{\prime}$ vertices, then $n=[k(\Delta-2)+2] n^{\prime}+k$. We obtain $n<2 t\left[n^{\prime}+1 /(\Delta-2)\right]$, and hence $t(G)>1 /(n / 2) /\left[n^{\prime}+1 /(\Delta-2)\right]$.

It remains to construct a suitable $H$ with $n^{\prime}$ as small as possible. When $\Delta=3$, we form $H$ by subdividing one edge of $K_{3,3}$; here $n^{\prime}=7$ and $t(G)>\frac{1}{8}(n / 2)$. For larger $\Delta$, consider the lexicographic composition $F_{s}=C_{5}\left[\bar{K}_{s}\right]$, expanding each vertex of a 5-cycle into an independent set of size $s$. The graph $F_{s}$ is $2 s$-regular and triangle-free, and for $s \geq 2$ it has a pair of easily described edge-disjoint Hamiltonian cycles. When $(\Delta+1) / 2$ is odd, we form $H$ by deleting from $F_{(\Delta+1) / 2}$ the odd-indexed edges on one Hamiltonian cycle. Since $n^{\prime}=2.5(\Delta+1)$ is odd, this reduces one vertex degree from $\Delta+1$ to $\Delta-1$ and the others to $\Delta$. When $(\Delta+1) / 2$ is even, we form $H$ by deleting from $F_{(\Delta+3) / 2}$ the odd-indexed edges on one Hamiltonian cycle and all edges on another Hamiltonian cycle. Since $n^{\prime}=2.5(\Delta+3)$ is odd, we again obtain the desired degree sequence. Here $\Delta \geq 7$, which yields the 7.7 in the statement of the result.

\section{THE MAIN RESULT}

We consider an $n$-vertex graph $G$ with maximum degree $\Delta$ and wish to prove that $I(G) \leq$ $(\Delta+1 / \Delta) n / 2$. We may assume that $G$ has no isolated vertices, because such vertices require no intervals; when $f(v)=\emptyset$ and the intersection graph is taken, $v$ becomes an isolated vertex.

Our approach is to select a set of edge-disjoint trails $T_{1}, \ldots, T_{k}$ to cover $E(G)$, in a greedy manner subject to various conditions; each new trail contains some previously uncovered edge. We partition $E(G)$ into sets associated with the trails; the set $E_{i}$ associated with $T_{i}$ consists of $E\left(T_{i}\right)$ and the newly covered edges that do not belong to later trails. We will also associate a set $S_{i} \subseteq V(G)$ with each trail (the union of closed neighborhoods of certain vertices of the trail); these sets will be pairwise disjoint. We can represent $E_{i}$ using $\left|E_{i}\right|+1$ intervals, fewer if $E_{i}$ contains a triangle with at most one edge on $T_{i}$.

When $G$ has maximum degree $\Delta$, we have $e(G) \leq \Delta n / 2$. If $e(G)=\Delta n / 2-k$, we will use $\left|E_{i}\right|+1$ intervals for at most $k+n /(2 \Delta)$ trails $T_{i}$. We do this by ensuring that we use an extra interval for $T_{i}$ only when there exists $\alpha_{i} \in\{0,1,2\}$ such that $\left|S_{i}\right| \geq\left(2-\alpha_{i}\right) \Delta$ and the degrees of two new vertices of $T_{i}$ sum to at most $2 \Delta-\alpha_{i}$. If the values of $\alpha_{i}$ over the $s$ trails using an extra interval sum to $r$, then we have $I(G) \leq e(G)+s$ and $e(G) \leq \Delta n / 2-r / 2$. Hence $I(G) \leq \Delta n / 2+(2 s-r) / 2$. Also we have associated $(2 s-r) \Delta$ vertices with these trails. Since $(2 s-r) \Delta \leq n$, we have the desired bound. The remainder of the proof consists of showing that we can choose the trails to ensure these conditions.

We use "open" trails; these are trails with two distinct endpoints. We say that a trail is closable if its endpoints are adjacent via an edge not belonging to the trail. When $T$ is closable, we let $T^{\prime}$ denote the closed trail formed by adding the edge between the endpoints of $T$.

Theorem. Every simple graph with $n$ vertices and maximum degree $\Delta$ has total interval number at most $(\Delta+1 / \Delta) n / 2$. Furthermore, equality holds only when every component is $K_{\Delta, \Delta}$. 
Proof. We select a sequence of pairwise edge-disjoint open trails $T_{1}, \ldots, T_{k}$ in a greedy manner. The new vertices of $T_{i}$ are the vertices in $T_{i}$ that do not appear in $T_{1}, \ldots, T_{i-1}$ and that cover at least one edge not covered by vertices of earlier trails. The new edges of $T_{i}$ are the previously uncovered edges that are covered by new vertices of $T_{i}$. Note that an edge of $T_{i}$ is new (for $T_{i}$ ) if and only if both its endpoints are new.

We choose each $T_{i}$ to be an open trail having endpoints that are new. Among these, we choose $T_{i}$ with maximum number of new vertices. Among these, we choose $T_{i}$ to be closable if such a candidate is available. Among the remaining candidates for $T_{i}$, we choose $T_{i}$ with minimum length.

The sequence ends when all edges are covered. The set $E_{i}$ of edges associated with $T_{i}$ is $E\left(T_{i}\right)$ together with the new edges that do not belong to later trails. By construction, these edge sets are disjoint. We postpone the definition of the vertex set $S_{i}$ associated with $T_{i}$.

Claim 1. If $T_{i}$ is not closable and has endpoint $v$, then only one edge incident to $v$ belongs to $T_{i}$. Otherwise, we delete the initial portion of $T_{i}$ up to the next appearance of a new vertex other than the other endpoint of $T_{i}$ (this may be $v$ again). The shorter trail $T$ is open and has the same new set as $T_{i}$; it may be closable, but since $T_{i}$ is not closable, we would in either case choose $T$ in preference to $T_{i}$.

Claim 2. If the vertices of $T_{i}$ are not all new, then $T_{i}$ is not closable and the end edges of $T_{i}$ are new. Let $x$ be the first vertex of $T_{i}$ that is not new, belonging to an earlier trail $T_{j}$. If $T_{i}$ is closable, then $T_{j}$ can absorb the closed trail $T_{i}^{\prime}$ to enlarge its new set. If $T_{i}$ is not closable, then the first edge of $T_{i}$ is new unless it is $u x$. By the maximality of the new set, every neighbor of $u$ along a new edge belongs to $T_{i}$. If $v$ is the first such vertex on $T_{i}$, then the $u, v$-portion of $T_{i}$ together with the edge $u v$ forms a closed trail containing $x$ that can be absorbed by $T_{j}$ to enlarge its new set.

Claim 3. If $T_{i}$ is closable, then no vertex of $T_{i}$ appears in another trail or has a neighbor in a later trail. By Claim 2, every vertex of $T_{i}$ is new. If $w$ is a vertex of $T_{i}$ that equals or is adjacent to a vertex $w^{\prime}$ of a later trail $T_{j}$, then we can traverse $T_{i}^{\prime}$ starting at $w$, enter $T_{j}$ at $w^{\prime}$, and continue to an end of $T_{j}$, replacing $T_{i}$ by a trail with at least two more new vertices.

Claim 4. If $T_{i}$ is not closable, then there is no edge to a later trail from an endpoint of $T_{i}$ or from its neighbor along $T_{i}$. Any such edge permits an extension of $T_{i}$ (or of $T_{i}$ minus its endpoint) using a portion of $T_{j}$ that has at least two new vertices, thereby creating a trail with more new vertices than $T_{i}$.

The start vertices of $T_{i}$ are its endpoints if $T_{i}$ is not closable, or all of its vertices if $T_{i}$ is closable. By Claim 3, every start vertex of $T_{i}$ is a new vertex of $T_{i}$.

Claim 5. No vertex of $T_{i}$ is adjacent to two start vertices of later trails, or to a start vertex of $T_{i}$ and a start vertex of a later trail. Suppose $w \in V\left(T_{i}\right)$ has neighbors $x, y$ that are start vertices of $T_{j}, T_{k}$, respectively, with $i \leq j \leq k$ and $i \neq k$. By Claim $3, T_{i}$ is not closable. By the "newness" of start vertices (and by Claim 4 if $i=j$ ), wx, wy do not belong to $E\left(T_{i}\right)$. If $j=k, T_{i}$ could thus absorb a portion of $T_{j}$ that contains a new vertex, giving $T_{i}$ more new vertices. Hence we may assume $i \leq j<k$. In this case, $w y \notin E\left(T_{j}\right)$, since $y$ is new in $T_{k}$. If $T_{j}$ is closable, then $j>i$ and Claim 3 yields $w x \notin E\left(T_{j}\right)$. If $T_{j}$ is not closable, then Claim 1, Claim 4 and the edge $w y$ imply that $w x \notin E\left(T_{j}\right)$. Now $T_{j}$, which we can view as ending at $y$, can be extended via $w$ to absorb at least two new vertices from $T_{k}$.

Claim 6. If $u, v$ are start vertices of $T_{i}, T_{j}$ with $i<j$, then $u, v$ are nonadjacent and have no common neighbor. By Claims 3 and 4 , a start vertex of $T_{i}$ has no neighbor in a later trail. No start vertex of $T_{i}$ has a neighbor outside all trails, because such a neighbor could be used to enlarge the new set of $T_{i}$. By Claim 5, $u$ and $v$ have no common neighbor in trail $T_{i}$ or earlier. 
Claim 7. If $E_{i}$ contains a triangle with at most one edge on $T_{i}$, then $E_{i}$ can be represented using $\left|E_{i}\right|$ intervals. If the vertices of $T_{i}$ are $v_{1}, \ldots, v_{n}$ in order (with repetition), then we represent $T_{i}$ by assigning the interval $\left(j-\frac{2}{3}, j+\frac{2}{3}\right)$ to $v_{j}$. This uses $e\left(T_{i}\right)+1$ intervals. For each additional edge $e \in E_{i}$ that is not in the triangle, suppose $e=x v_{j}$ where $v_{j}$ is a new vertex of $T_{i}$. We represent $e$ by adding a small interval for $x$ within $\left(j-\frac{1}{3}, j+\frac{1}{3}\right)$ (intersecting only the interval for $v_{j}$ ). If the triangle in $E_{i}$ contains an edge $v_{j} v_{j+1}$ of $T_{i}$, then we add an interval for their common neighbor in $E_{i}$ within $\left(j+\frac{1}{3}, j+\frac{2}{3}\right)$, gaining two edges for one interval. If it contains no edge of $T_{i}$, we select some $v_{j} \in V\left(T_{i}\right)$ on the triangle and add a common interval for the other two vertices of the triangle within $\left(j-\frac{1}{3}, j+\frac{1}{3}\right)$, gaining three edges for two intervals. In total, we have used $\left|E_{i}\right|$ intervals.

Having proved these claims, we let $S_{i}$ consist of the start vertices of $T_{i}$ and their neighbors. By Claim 6, the sets $S_{i}$ are pairwise disjoint. Choose two start vertices $u, v$ in $T_{i}$ with the minimum degree sum. If $d(u)+d(v) \leq 2 \Delta-2$, then by the discussion before the theorem statement we do not need to save an interval for $T_{i}$. If $d(u)+d(v)=2 \Delta-1$, then one of $u, v$ has degree $\Delta$ and we have $\left|S_{i}\right|>\Delta$.

Hence we may assume that every start vertex of $T_{i}$ has degree $\Delta$. By the computation before the theorem statement, it remains only to show that $\left|S_{i}\right| \geq 2 \Delta$ if $E_{i}$ does not contain a triangle with at most one edge on $T_{i}$. If some pair of start vertices on $T_{i}$ has no common neighbor, then $\left|S_{i}\right| \geq 2 \Delta$, so we may assume that every pair of start vertices has a common neighbor.

Suppose first that $T_{i}$ is not closable. Let $u, v$ be the endpoints of $T_{i}$, and let $w$ be a common neighbor; by Claim 5, $w$ does not belong to an earlier trail. If neither of $\{u w, v w\}$ belongs to $T_{i}$, then $T_{i}$ can be extended by $u w$ to obtain a closable trail with the same new set as $T_{i}$, which would be preferred to $T_{i}$. This includes the case where $u, v$ are adjacent and $T_{i}$ has length 1 . In the remaining case, $u, v$ are nonadjacent and any common neighbor of them is adjacent to one of them using an end edge of $T_{i}$. There are at most two such common neighbors. Hence $\left|S_{i}\right| \geq 2+2 \Delta-2=2 \Delta$, as desired.

Finally, suppose that $T_{i}$ is closable, which requires at least three vertices, each pair of which has a common neighbor. By Claims 3 and 5, the common neighbors of vertices in $T_{i}$ also lie in $T_{i}$. Furthermore, every edge of $T_{i}^{\prime}$ forms a triangle only using two other edges of $T_{i}^{\prime}$; otherwise, we can use the endpoints of that edge as the endpoints of $T_{i}$ and use the common neighbor to form a triangle having at most one edge on $T_{i}$.

Since $T_{i}^{\prime}$ forms a connected subgraph of $G$, it has a vertex $w$ that is not a cut-vertex of $T_{i}^{\prime}$. Deleting from $T_{i}^{\prime}$ any set of edges incident to $w$ leaves a connected subgraph, except possibly for isolating $w$. Every edge $w v$ incident to $w$ in $T_{i}^{\prime}$ lies on a triangle in $T_{i}^{\prime}$; let $u$ be a third vertex of this triangle. Deleting $\{w v, w u\}$ from $T_{i}^{\prime}$ leaves a subgraph having a $u, v$-Eulerian trail $T$. Now $u, v, w$ form a triangle with only one edge on $T$. Furthermore, every edge of $E_{i}$ is incident to at least one vertex of $T$, because when $T_{i}$ is closable every edge of $E_{i}$ has both endpoints on $T_{i}$. By the construction in Claim 7, we can represent $E_{i}$ using only $\left|E_{i}\right|$ intervals, saving one for the edges $\{w v, w u\}$.

We have resolved all cases, and the proof of the bound is complete. Next we consider how equality may be achieved. We may assume that $G$ is connected. It suffices to show that if $G \neq K_{\Delta, \Delta}$, then we save an extra interval for $T_{1}$.

If $T_{1}$ is closable, then Claim 3 implies that $V\left(T_{1}\right)=V(G)$, and hence $E_{1}=E(G)$. If $G$ is not $\Delta$-regular or if $n>2 \Delta$, then $|E(G)|+1<(\Delta+1 / \Delta) n / 2$, and we are done. If $G$ is $\Delta$-regular and $\Delta \geq n / 2$, then $G$ is Hamiltonian, by Dirac's Theorem. If $G \neq K_{\Delta, \Delta}$, then $G$ has a triangle, by Turán's Theorem. By the choice of $T_{1}$ to minimize length, $T_{1}^{\prime}$ is a Hamiltonian cycle. If $T_{1}^{\prime}$ uses any edge of the triangle, then we delete that edge from $T_{1}^{\prime}$ to obtain $T_{1}$. Hence 
we can choose $T_{1}$ so that we have a triangle with at most one edge on $T_{1}$. By Claim 7, we can now represent $E(G)$ using $|E(G)|=\Delta n / 2$ edges.

If $T_{1}$ is not closable, recall the computation of our bound on $I(G)$. We have $I(G) \leq \Delta n / 2+$ $(2 s-r) / 2$, where there are $s$ trails $T_{i}$ using $\left|E_{i}\right|+1$ intervals and $r=\sum \alpha_{i}$, with $2 \Delta-\alpha_{i}$ being the sum of the degrees of the chosen vertices on $T_{i}$. We proved the bound by associating $(2 s-r) \Delta$ vertices with these trails, since then $(2 s-r) \Delta \leq n$. We have strict inequality if some $T_{i}$ uses only $\left|E_{i}\right|$ intervals (since its start vertices are not in the sets $S_{j}$ associated with other trails) or if for some $T_{i}$ the associated set $S_{i}$ has more than $2 \Delta-\alpha_{i}$ vertices.

Now consider $T_{1}$, with endpoints $u, v$. If $d(u)+d(v)<2 \Delta$, then $\left|S_{i}\right|>\left(2-\alpha_{i}\right) \Delta$ immediately, so we may assume $d(u)+d(v)=2 \Delta$. By Claim 4, all neighbors of $v$ lie in $T_{1}$. By Claim 1 , only one edge incident to $v$ belongs to $T_{1}$. Since our greedy selection prefers closable trails, this implies that $u, v$ are not adjacent (unless $u v=E\left(T_{1}\right)$, in which case $\Delta=1$ and $G=K_{\Delta, \Delta}$ ). Suppose the vertices of $T_{1}$ are $u=x_{1}, x_{2}, \ldots, x_{m}=v$ in order. If $v$ is adjacent to both $x_{i}$ and $x_{i+1}$ for some $i<m-2$, then it forms a triangle with one edge on $T_{i}$, and Claim 7 applies. If $i=m-2$, then because $v$ has no neighbors on later trails, we can again save an interval for this triangle. Hence we may assume that $v$ does not have consecutive neighbors on $T_{i}$. If $v$ is adjacent to $x_{i}$ for $i<m-1$, then $x_{i+1}$ has no neighbor $w$ in another trail $T_{j}$. Otherwise, we could follow $x_{1}, \ldots, x_{i}, v, x_{m-1}, \ldots, x_{i+1}, w$ and continue in $T_{j}$ to enlarge the new set of $T_{1}$. Since the successors on $T_{1}$ of neighbors of $v$ have no neighbors in later trails, they appear in no later $S_{j}$, and we can add them to $S_{1}$. Now $S_{1}$ consists of at least the neighbors of $v$, their successors on $T_{1}$, and $u$, which totals $2 \Delta+1$ vertices.

The alterations that are used to improve trails always increase the new set or decrease the length (or make it closed); there can be at most $n^{2}$ of these changes for each trail. Also the search for whether a change is needed takes polynomial time. Hence this proof can be implemented as a polynomial algorithm to produce a representation that satisfies the bound.

\section{References}

[1] M. Aigner and T. Andreae, The total interval number of a graph, J. Comb. Theory (B) 46 (1989), 7-21.

[2] J. R. Griggs and D. B. West, Extremal values of the interval number of a graph, I, SIAM J. Algeb. Disc. Meth. 1 (1980), 1-7.

[3] A. V. Kostochka, in Abstracts of 8th All-Union Conference on Theoretical Cybernetics, Gorkii, Part 1 1988, p. 174 (in Russian).

[4] T. M. Kratzke, The total interval number of a graph. Ph.D. Thesis, Univ. of Illinois (1987), Coordinated Science Laboratory Research Report UILU-ENG-88-2202.

[5] T. M. Kratzke and D. B. West, The total interval number of a graph I: Fundamental classes, Discrete Math. 118 (1993), 145-156.

[6] T. M. Kratzke and D. B. West, The total interval number of a graph II: Trees and complexity, SIAM J. Discr. Math. 9 (1996), 339-348.

[7] W. T. Trotter and F. Harary, On double and multiple interval graphs, J. Graph Theory 2(1978), 137-142. 